\title{
The effect of anisotropy on the propagation of linear compressional waves in magnetic flux tubes: Applications to astrophysical plasmas
}

\author{
I. Ballai ${ }^{1}$ and A. Marcu ${ }^{2}$ \\ 1 Space \& Atmosphere Research Center (SPARC), Dept. of Applied Mathematics, University of Sheffield, Hicks Building, \\ Hounsfield Road, Sheffield, S3 7RH, UK \\ 2 Babes-Bolyai University, Dept. of Theoretical Physics, 1 Kogalniceanu, 3400-Cluj Napoca, Romania
}

Received 24 June 2003 / Accepted 6 November 2003

\begin{abstract}
The propagation of linear compressional waves is studied in magnetic flux tubes. The plasma inside and outside the tube is considered to be rarefied which confers an anisotropic character to the plasma. The anisotropy appears at different temperatures for parallel and perpendicular directions relative to the ambient magnetic field. The theoretical results are applied to study the effects of anisotropy and equilibrium steady flow on wave propagation in structures such as coronal plumes, the geomagnetic tail and anisotropic astrophysical jets. The obtained results show that the internal and/or external anisotropy has a very important effect on the propagation of linear compressional waves. The presence of a steady equilibrium flow in certain applications can change dramatically the propagation of the modes.
\end{abstract}

Key words. plasmas - Sun: solar wind - waves - magnetic fields - ISM: jets and outflow

\section{Introduction}

One of the most interesting processes in solar and astrophysical plasmas is the complicated interaction of plasma motions with magnetic fields. These media are highly non-uniform and, as a consequence, are a natural environment for magnetohydrodynamic (MHD) waves. Interest in these waves has proceeded for a variety of reasons. First, it is likely that the waves which have been observed in the solar atmosphere and interplanetary space significantly influence the thermal and dynamic properties of the plasma. Waves can transport energy and momentum. When part of their energy or momentum is transferred to the plasma they can heat and accelerate the plasma. In this context, waves have been used to tackle the complicated problem of coronal heating (resonant absorption, resonant waves, phase-mixing, ion-cyclotron resonance, etc.) and to explain the acceleration of the fast solar wind. Waves are also of direct interest to us since it is known that phenomena such as geomagnetic storms, upper atmospheric and ionospheric heating, aurorae and even our weather are related to the thermal and dynamic states of the solar corona and solar wind and also to the waves themselves as they impinge on the Earth's magnetosphere. Second, some of the waves observed far from the Sun appear to have their origin at the Sun. Therefore, they represent valuable probes of physical processes occurring in the solar

Send offprint requests to: I. Ballai,

e-mail: i.ballai@sheffield.ac.uk atmosphere, i.e. plasma diagnostic. This is based on the remarkable property of waves to carry information about the plasma in which they occur. Finally, waves in space plasmas represent a valuable tool for studying the behaviour of magnetic astrophysical plasmas, the characteristics of which cannot be reproduced under laboratory conditions.

The derivation of the isotropic MHD equations requires the assumption that electrons are tightly coupled to ions, so that plasma temperatures are isotropic. In this case the plasma density and temperature satisfy some equation of state. However, spacecraft observations (Kohl et al. 1998) often show anisotropic velocity and temperature distributions, implying that this model is not fully valid for many space plasmas (for example, the solar wind can be considered as a collisionless medium beyond $r=1.5 R_{\odot}$ ). In this kind of plasma, different types of particle can have different temperatures parallel and perpendicular to the ambient magnetic field. Thus, more complex models based on more sophisticated closure relations may be necessary to model adequately the rich diversity of plasma dynamics.

Parker (1958) was the first to point out that the interplanetary magnetic field might lead to an anisotropic pressure tensor. When the cyclotron frequency of the ions is much higher than the collisional frequency, the particles gyrate many times around a line of magnetic force between two collisions. The magnetic field induces a splitting in the pressure, introducing a parallel and a perpendicular component. The two components 
of the pressure are not necessarily equal. However, the thermal anisotropy and electron conduction velocity relative to the ions generally excite plasma oscillations that scatter the particles, pulling the plasma toward isotropy, $\left(p_{\|} \sim p_{\perp}\right)$.

The main complication arising from the presence of anisotropy relates to pitch angle scattering, a mostly kinetic effect. Such a description within fluid approximation remains a fundamental but unresolved theoretical problem. Technically speaking, the closure of the fluid equations requires two equations of state, one for the parallel and one for the perpendicular pressure. In spite of much effort, there is no generally accepted form for these two equations of state. In such a medium waves that are mostly generated by the free energy of the particle distribution functions are the means to relax this free energy. Waves, once amplified, can heat particles, permit exchange of energy between different populations of particles and precipitate particles.

An original approach to the problem of physical properties of rarefied plasmas has been the theory developed by Chew et al. (1956) known as the Chew-Goldberger-Low (CGL) approximation. The usual MHD equations are derived from the Boltzmann equation using an expansion in powers of the collisional mean free path. In this case the plasma is collisiondominated therefore the collisional term in the Boltzmann equation is the leading term, with all other terms being treated as perturbations. When the density is so small that the plasma can be considered as a collisionless medium, a new form of governing equations can be derived from the Boltzmann equation using an expansion in powers of the Larmor radius. This approximation can be considered as an adiabatic approximation since it depends on the Larmor frequency being large compared to other characteristic frequencies of the problem.

The properties of anisotropic plasmas have been studied intensively over the past few years in the light of new observations (see, e.g. Kato et al. 1966; Hau \& Lin 1995; Nakariakov \& Oraevsky 1995; Ballai et al. 2002a,b). These studies revealed that the properties of the waves are different from the usual MHD and these differences are crucial from the wave propagation and stability point of view. Since the study of compressional waves in anisotropic plasmas is in its infancy there is no general concept as to how these waves are generated. However, it is very likely that the generation mechanism of these waves in similar to the mechanisms that generate the well-known MHD (isotropic) waves: convection, reconnection, resonances with other modes, even non-anisotropic, etc. A wave that propagates in the isotropic plasma "decays" into a two-component wave (i.e. parallel and perpendicular sound speeds) since in the high corona and beyond, observations show that the plasma's temperature has different values measured parallel and perpendicular to the direction of the magnetic field.

Space and ground-based telescopes have revealed that the space plasma is dynamic, showing a steady equilibrium flow on all spatial and temporal scales along or nearly along the magnetic field lines. The presence of an equilibrium flow in an isotropic or anisotropic plasma can alter many processes and can introduce new effects, such as negative energy waves, Kelvin-Helmholtz instabilities, resonant flow instabilities, etc. (see, e.g. McKenzie 1970; Srivastava 1977;
Trussoni et al. 1988; Nakariakov \& Roberts 1995; Joarder 2002; Taroyan \& Erdélyi 2003; Homem et al. 2003). In our treatment, the presence of an equilibrium steady flow plays a key role and the propagation of compressional waves is studied with respect to the strength of the plasma flow.

The paper is organized as follows. In Sect. 2 we introduce the basic equations used in the present paper, emphasizing the differences between the usual MHD and the rarefied plasma description. Section 3 is devoted to the study of the waves that appear in solar and astrophysical structures (coronal plumes, geomagnetic tail, anisotropic astrophysical jets) using a double-polytropic pressure law. In order to show the importance of the anisotropy and equilibrium flow, we plot the variation of the phase speed for linear compressional waves with respect to these two effects for thin and thick flux tubes. Finally, in Sect. 4 we summarize and discuss our results.

\section{Basic equations}

Let us consider a magnetic flux tube of infinite extension in the $z$ direction embedded in a magnetic plasma. The plasma inside (with density $\rho_{0}$ ) and outside (with density $\rho_{\mathrm{e}}$ ) the tube is considered anisotropic. The density varies only in the transverse direction, being uniform inside and outside the tube and having a discrete jump at the duct's boundary. Several simplifying assumptions are made including the neglect of any dissipative effect and gravity. The insertion of this duct (waveguide) introduces a new length-scale in addition to the wavelength, the fundamental length-scale of the waves.

The plasma motion is described by the ideal single-fluid MHD equations where a double-polytropic pressure law is used (Hau \& Lin 1995)

$$
\begin{aligned}
& \frac{D \rho}{D t}+\rho \nabla \cdot \boldsymbol{v}=0, \quad \nabla \cdot \boldsymbol{B}=0, \\
& \rho \frac{D \boldsymbol{v}}{D t}=-\nabla \cdot \boldsymbol{P}+\frac{1}{\mu}[(\nabla \times \boldsymbol{B}) \times \boldsymbol{B}], \\
& \frac{\partial \boldsymbol{B}}{\partial t}=\nabla \times(\boldsymbol{v} \times \boldsymbol{B}), \\
& \frac{D}{D t}\left(\frac{p_{\perp}}{\rho B^{\gamma_{\perp}-1}}\right)=0, \quad \frac{D}{D t}\left(\frac{p_{\|} B^{\gamma_{\|}-1}}{\rho^{\gamma_{\|}}}\right),
\end{aligned}
$$

where $D / D t=\partial / \partial t+\boldsymbol{v} \cdot \nabla$ is the convective operator, $\gamma_{\|}, \gamma_{\perp}$ are the parallel and perpendicular polytropic indices and $\rho, \boldsymbol{B}$, $\boldsymbol{v}, p_{\|}$and $p_{\perp}$ denote the density, magnetic field, velocity and the parallel and perpendicular component of the pressure. The polytropic indices express the increase in temperature upon plasma compression. For $\gamma_{\perp}=2$ and $\gamma_{\|}=3$ the double adiabatic CGL equations are recovered whereas for $\gamma_{\|}=\gamma_{\perp}=1$ we obtain the isothermal limit. The use of this system is fully supported observationally. Recently Farrugia et al. (2001) have shown that near the subsolar region the particles obey double polytropic laws of indices 1 and 1.5 in the perpendicular and parallel direction, respectively. The system (1)-(4) is a particular case of the most general polytropic laws introduced by Abraham-Shrauner (1967). 
In the equation of momentum conservation (Eq. (2)), $\boldsymbol{P}=$ $p_{\perp} \hat{\mathbf{I}}+\left(p_{\|}-p_{\perp}\right) \boldsymbol{b} \boldsymbol{b}$ is the pressure tensor, where $\hat{\mathbf{I}}$ is the unit dyadic and $\boldsymbol{b}=\boldsymbol{B} / \boldsymbol{B}$ is the unit vector parallel to the magnetic field direction.

In the system of Eqs. (1)-(4) we write all the quantities as $f_{0}+f$ where $f_{0}$ are the equilibrium quantities and $f$ their Eulerian perturbations. We suppose a $\boldsymbol{B}_{0}=B_{0} z$ equilibrium magnetic field with $\rho_{0}, p_{\|}^{(0)}, p_{\perp}^{(0)}$ the equilibrium density and parallel and perpendicular kinetic pressures of the plasma. We consider small disturbances around the equilibrium values so, all products or squares of perturbed quantities can be neglected. The perturbations can be Fourier-analyzed with respect to the $\varphi$ and $z$ coordinates. Perturbations oscillate with the same real frequency, $\omega$, so they are of the form $\sim \exp [\mathrm{i} \omega t+\mathrm{i} m \varphi+\mathrm{i} k z]$. Obviously, $m$ has to be an integer and $k$ real. Without loss of generality we may assume that $m$ is positive. The expanded system of equations is given in the Appendix.

The system of linearized MHD Eqs. (A.1)-(A.8) can be reduced to a system of two coupled ODE for the perpendicular component of the total pressure perturbation and the radial component of the velocity

$\frac{\mathrm{d} P_{\perp}}{\mathrm{d} r}=\frac{\mathrm{i} \rho_{0}}{\omega} D_{\mathrm{A}} v_{\mathrm{r}}$,

$\left[\frac{1}{D_{\mathrm{C}}}\left(\omega^{2}-k^{2} c_{\mathrm{s} \|}^{2}\right)-\frac{m^{2}}{r^{2}} \frac{1}{D_{\mathrm{A}}}\right] P_{\perp}=-\frac{\mathrm{i} \rho_{0}}{\omega} \frac{1}{r} \frac{\mathrm{d}}{\mathrm{d} r}\left(v_{\mathrm{r}} r\right)$,

where the coefficients $D_{\mathrm{A}}$ and $D_{\mathrm{C}}$ are given by

$D_{\mathrm{A}}=\omega^{2}-k^{2} v_{\mathrm{A}}^{2}(1-\Gamma)=\omega^{2}-k^{2} v_{\mathrm{A}}^{* 2}$,

$D_{\mathrm{C}}=\left(v_{\mathrm{A}}^{2}+c_{\mathrm{S} \perp}^{2}\right)\left(\omega^{2}-k^{2} c_{\mathrm{T}}^{2}\right)$,

and

$c_{\mathrm{T}}^{2}=\frac{v_{\mathrm{A}}^{2} c_{\mathrm{S} \|}^{2}+c_{\mathrm{S} \perp}^{2}\left(c_{\mathrm{S} \|}^{2}-\frac{c_{\mathrm{S} \perp}^{2}}{\gamma_{\perp}^{2}}\right)}{v_{\mathrm{A}}^{2}+c_{\mathrm{S} \perp}^{2}}$.

Compared to the results obtained in an isotropic collisional plasma (see, e.g. Edwin \& Roberts 1983) the Alfvén and cusp $\left(c_{\mathrm{T}}\right)$ speeds are modified by the anisotropy. A detailed study of the properties of Alfvén and slow magnetoacoustic waves propagating in an unbounded medium can be found in, e.g. Ballai et al. (2002b). In order to make our analysis clearer we must recall shortly some of the properties of these waves.

First of all, Alfvén waves propagate with the modified phase speed $v_{\mathrm{A}}^{*}=v_{\mathrm{A}}(1-\Gamma)^{1 / 2}$. When $\Gamma=0$ (isotropic case) we recover the usual Alfvén speed. Whereas the velocity in the isotropic case is always positive definite, $(1-\Gamma)$ may be negative $\left(p_{\|}^{(0)}>p_{\perp}^{(0)}+B^{2} / \mu\right)$. In the later case, the Alfvén mode will exhibit non-propagating, pure exponential growth. This instability, called the firehose instability, depends on the bulk properties of the plasma, and thus is non-resonant. It appears when the destabilizing centrifugal force exerted by particle motion along the magnetic field lines exceeds the restoring force associated with tension in the magnetic field lines. Inspecting the expression of the anisotropy factor, $\Gamma$, we can see that we have to consider the contribution of all three pressures $\left(p_{\|}^{(0)}\right.$, $p_{\perp}^{(0)}$ and $\left.B_{0}^{2} / 2 \mu\right)$ when considering Alfvén waves.
Slow waves propagate with a modified phase speed given by (6). For the isotropic case (i.e. when $c_{\mathrm{S} \perp}^{2}=c_{\mathrm{S} \|}^{2}=c_{\mathrm{S}}^{2}$ and $\left.\gamma_{\perp}=1\right) c_{\mathrm{T}}$ reduces to the classical cusp speed which is always positive. However, in anisotropic plasmas the cusp speed can be negative for $c_{\mathrm{S} \|}^{2}<c_{\mathrm{S} \perp}^{4} /\left[\gamma_{\perp}^{2}\left(v_{\mathrm{A}}^{2}+c_{\mathrm{S} \perp}^{2}\right)\right]$ and this condition gives rise to the mirror instability threshold. If this condition is satisfied, the magnetic field develops regions of low field strength separated by regions of enhanced field strength parallel to the equilibrium magnetic field direction. Where the field is stronger than the unperturbed field, the particle mirror points shift in such a way that the plasma density decreases. However, in the present study we shall avoid the appearance of the two instabilities mentioned above.

We can easily see that the cusp speed exceeds the Alfvén speed (reversible MHD) only if

$\beta_{\|} \gamma_{\|}>\frac{2 \beta_{\perp}^{2}}{\beta_{\perp} \gamma_{\perp}+2}$

where $\beta_{\|}=2 c_{\mathrm{S} \|}^{2} /\left(\gamma_{\|} v_{\mathrm{A}}^{2}\right)$ and $\beta_{\perp}=2 c_{\mathrm{S} \perp}^{2} /\left(\gamma_{\perp} v_{\mathrm{A}}^{2}\right)$ are the plasma- $\beta$ parameters in parallel and perpendicular directions.

Let us now discus waves in a cylindrical waveguide. The equilibrium configuration of the plasma inside and outside the magnetic tube is characterized by

$\begin{cases}B_{0}, p_{\perp}^{(0)}, p_{\|}^{(0)}, \rho^{(0)}, & |r|<R \\ B_{\mathrm{e}}, p_{\|}^{(\mathrm{e})}, p_{\perp}^{(\mathrm{e})}, \rho^{(\mathrm{e})}, & |r|>R\end{cases}$

where the superscripts " 0 " and " $\mathrm{e"} \mathrm{denote} \mathrm{quantities} \mathrm{inside} \mathrm{and}$ outside the slab. Convenient notation for later use are the sound speeds and the two Alfvén speeds outside and inside the tube defined as

- the external sound speeds in the parallel and perpendicular direction relative to the ambient magnetic field

$$
c_{\mathrm{e} \|}^{2}=\frac{\gamma_{\|} p_{\|}^{(\mathrm{e})}}{\rho^{(\mathrm{e})}}, \quad c_{\mathrm{e} \perp}^{2}=\frac{\gamma_{\perp} p_{\perp}^{(\mathrm{e})}}{\rho^{(\mathrm{e})}} ;
$$

- the internal sound speeds in the parallel and perpendicular direction relative to the ambient magnetic field

$$
c_{0 \|}^{2}=\frac{\gamma_{\|} p_{\|}^{(0)}}{\rho^{(0)}}, \quad c_{0 \perp}^{2}=\frac{\gamma_{\perp} p_{\perp}^{(0)}}{\rho_{0}^{(0)}} ;
$$

- the Alfvén speeds outside and inside the tube

$$
v_{\mathrm{Ae}}^{2}=\frac{B_{\mathrm{e}}^{2}}{\mu \rho^{(\mathrm{e})}}, \quad v_{\mathrm{A} 0}^{2}=\frac{B_{0}^{2}}{\mu \rho^{(0)}} .
$$

The pressure balance across the tube implies that the total pressure in the normal direction to the duct's wall is constant, so

$p_{\perp}^{(\mathrm{e})}+\frac{B_{\mathrm{e}}^{2}}{2 \mu}=p_{\perp}^{(0)}+\frac{B_{0}^{2}}{2 \mu}$

leading to a density contrast

$\mathcal{D}=\frac{\rho^{(\mathrm{e})}}{\rho^{(0)}}=\frac{2 c_{0 \perp}^{2}+\gamma_{\perp} v_{\mathrm{A} 0}^{2}}{2 c_{\mathrm{e} \perp}^{2}+\gamma_{\perp} v_{\mathrm{Ae}}^{2}}$ 
Using the system of Eq. (5), the equation that describes the evolution of the perpendicular component of the total pressure perturbation reads

$$
\frac{\mathrm{d}^{2} P_{\perp}}{\mathrm{d} r^{2}}+\frac{1}{r} \frac{\mathrm{d} P_{\perp}}{\mathrm{d} r}-\left(m_{0}^{2}+\frac{n^{2}}{r^{2}}\right) P_{\perp}=0,
$$

where the square of the magnetoacoustic parameter $m_{0}^{2}$ is defined as

$$
m_{0}^{2}=\frac{\left(k^{2} c_{0 \|}^{2}-\omega^{2}\right)\left(k^{2} v_{\mathrm{A} 0}^{* 2}-\omega^{2}\right)}{\left(c_{0 \perp}^{2}+v_{\mathrm{A}}^{2}\right)\left(k^{2} c_{\mathrm{T} 0}^{2}-\omega^{2}\right)} .
$$

Considering $\omega^{2}$ and $k^{2}$ real, $m_{0}^{2}$ can be positive or negative, i.e. the waveguide can support surface (non-oscillatory) or body (oscillatory) modes inside the slab. The perpendicular component of the total pressure perturbation can be expressed with the aid of the normal component of the velocity using Eq. (5).

In the external region the variation of the perpendicular component of the total pressure perturbation is described by an equation similar to Eq. (11), except that $m_{0}$ is replaced by an equivalently defined external $m_{\mathrm{e}}\left(v_{\mathrm{Ae}}, c_{\mathrm{e} \perp}, c_{\mathrm{e} \|}\right.$ are substituted for $v_{\mathrm{A} 0}$, etc.). Since we would like to focus on trapped (non-leaky) waves only, we suppose from the very beginning that $m_{\mathrm{e}}^{2}$ is positive. In the present context $m_{\mathrm{e}}^{-1}$ can be interpreted as the decay length of perturbations outside the cylinder. The above imposed restriction means that the amplitude of the waves decline exponentially with $r$ outside the tube, i.e. there is no appreciable disturbance. Bearing in mind the expression for $m_{\mathrm{e}}^{2}$, it is straightforward to show that in the external region the phase speed of the waves is either in the interval $\omega / k<c_{\mathrm{Te}}$ or $\min \left(c_{\mathrm{e} \|}, v_{\mathrm{Ae}}^{*}\right)<\omega / k<\max \left(c_{\mathrm{e} \|}, v_{\mathrm{Ae}}^{*}\right)$.

Equation (11) must be solved in the internal and external regions subject to the appropriate boundary conditions: the solutions must be regular on the axis and must decay exponentially at infinity. We also take into account the matching conditions. The interface between the two types of plasma (inside and outside the cylinder) is perturbed, so at this interface we have to impose the equality of the total pressure perturbation in the perpendicular direction and the constraint that the displacement of the interface is the same in the two regions. Consequently, we obtain that the dispersion relation for surface waves is given by

$$
\frac{\left(\omega^{2}-k^{2} v_{\mathrm{A0}}^{* 2}\right)}{\left(\omega^{2}-k^{2} v_{\mathrm{Ae}}^{* 2}\right)} m_{\mathrm{e}} \frac{K_{n}^{\prime}\left(m_{\mathrm{e}} R\right)}{K_{n}\left(m_{\mathrm{e}} R\right)}=\mathcal{D} m_{0} \frac{I_{n}^{\prime}\left(m_{0} R\right)}{I_{n}\left(m_{0} R\right)} .
$$

Following the same procedure, we obtain the dispersion relation for body waves as

$$
\frac{\left(\omega^{2}-k^{2} v_{\mathrm{A0} 0}^{* 2}\right)}{\left(\omega^{2}-k^{2} v_{\mathrm{Ae}}^{* 2}\right)} m_{\mathrm{e}} \frac{K_{n}^{\prime}\left(m_{\mathrm{e}} R\right)}{K_{n}\left(m_{\mathrm{e}} R\right)}=\mathcal{D} n_{0} \frac{J_{n}^{\prime}\left(n_{0} R\right)}{J_{n}\left(n_{0} R\right)},
$$

where $J_{n}$ is the Bessel function of the first kind, $I_{n}$ and $K_{n}$ are modified Bessel functions and the dash denotes the derivative of the Bessel functions with respect to their argument.

In what follows we pay attention only to the special cases corresponding to $n=0$ (cylindrically symmetric modes or sausage modes) and $n=1$ (asymmetric or kink modes). The sausage mode represents a propagating change in the crosssectional area, while the kink mode is a sideways displacement. The two dispersion relations obtained above have been previously derived for isotropic plasma by Wilson (1980), Spruit (1982), Edwin \& Roberts (1983) and references therein.

In the next section we solve the dispersion relations (Eqs. (13) and (14)) for given ordering of the characteristic phase speeds for particular cases found in solar and astrophysical plasmas.

\section{Ducted waves in anisotropic space plasmas}

\subsection{Ducted waves in coronal plumes}

Plumes are an intrinsic feature of polar coronal holes. They appear in white-light coronagraph and eclipse as ray-like structures, extending up to many solar radii above the solar limb. The study of coronal plumes became an important issue of the modern solar physics after they were observed in extreme ultraviolet (EUV) by SOHO and TRACE. While plumes may well not contribute significantly to the fast solar wind, they are major features of coronal holes. Plumes (together with their surrounding, the interplume region) require detailed theoretical modelling. They occur in magnetic field concentrations situated at the corners of the network cells.

Observational evidence of compressional waves in the solar wind has only been obtained recently. DeForest \& Gurman (1998), using high-cadence EIT/SOHO observations, found quasi periodic fluctuations with periods of $10-15 \mathrm{~min}$. in solar plumes, with a filamentary structure within the plume on a spatial scale of 3-5 arcsec. These fluctuations were identified as either sound or slow magnetoacoustic waves propagating along the plumes at $75-150 \mathrm{~km} \mathrm{~s}^{-1}$. Ofman et al. (2000) and Banerjee et al. (2001) detected quasi periodic variations in the polarization brightness at $1.9 R_{\odot}$ in both plume and interplume regions. Their Fourier power spectrum shows significant peaks around $1.6-2.5 \mathrm{mHz}$ and additional smaller peaks at longer and shorter time-scales. Their wavelet analysis of the polarization brightness time series shows that the coherence time of the fluctuations is about $30 \mathrm{~min}$. Recently, Banerjee et al. (2001) using $\mathrm{CDS} / \mathrm{SOHO}$ images detected long period slow magnetoacoustic waves in the solar inter-plume region which were produced at the network boundaries in the coronal hole.

Considering typical plume parameters $\left(T=1.6 \times 10^{6} \mathrm{~K}\right.$, $n=10^{8} \mathrm{~cm}^{-3}$ ), the condition of a collisionless plasma in the polar plume means that we consider waves with frequencies larger than the collisional frequency $\left(\omega_{\text {coll }} \approx 2.3 \mathrm{~Hz}\right)$, i.e. waves with periods smaller than $2.7 \mathrm{~s}$. Since this frequency is much larger than the observed frequencies in plumes, we can conclude that the effect of anisotropy is relevant to high-frequency waves which have not be yet observed.

We model the polar plume region with a radial, circular cross-section cylinder embedded in a coronal hole, the interplume region. We suppose the magnetic field is parallel to the $z$-axis of the tube and its value is similar inside and outside the tube $\left(B_{0}=B_{\mathrm{e}}\right)$. Since observations show that plumes are denser than the interplumes, we choose the density contrast (given by Eq. (10)) to be $1 / 5$. These enable us to conclude 
that $v_{\mathrm{Ae}}^{*}>v_{\mathrm{A} 0}^{*}$. In the plume and interplume regions sound speeds are much below the Alfvén speeds. This picture resembles the one discussed by Edwin \& Roberts (1983) about waves in coronal loops. Similar to their case, there are no surface waves, but there are body waves with phase speeds in the interval $\left(c_{\mathrm{T} 0}, c_{0 \|}\right)$ (slow body waves) and $\left(v_{\mathrm{A} 0}^{*}, v_{\mathrm{Ae}}^{*}\right)$ (fast body waves). We can see that slow body waves are limited only by internal quantities while fast waves appear in an interval bounded from above by the modified external Alfvén speed.

The dispersion relation given by Eq. (14) has a rich spectrum of solutions, and analytical treatment of these equations can be carried out only for special cases. In what follows, special attention is paid to the cases of slender (thin) and wide tubes corresponding to the long and short wavelength limits, respectively. The dependence of the propagation speed on the dimensionless quantity $k R$ for intermediate values is investigated numerically and is shown in Figs. 1 and 2.

In the long wavelength approximation the considered wavelengths are much longer than the width of the tube $\left(m_{0} R \ll 1\right.$ as $k R \ll 1$ ). Using the form of the Bessel functions for small arguments (Abramowitz \& Stegun 1967) we obtain that slow sausage body waves are propagating with the phase speed

$$
\begin{aligned}
\frac{\omega}{k} \approx & \pm c_{\mathrm{T}} \\
& \times\left\{1-\mathcal{D} \frac{\left(v_{\mathrm{Ae}}^{2 *}-c_{\mathrm{T} 0}^{2}\right) c_{0 \perp}^{4}}{4\left(c_{0 \perp}^{2}+v_{\mathrm{A} 0}^{2}\right)^{2} \gamma_{\perp}^{2} c_{\mathrm{T} 0}^{2}} \ln \left(\lambda_{\mathrm{p}}^{(1)}|k| R\right) k^{2} R^{2}\right\},
\end{aligned}
$$

where

$\lambda_{\mathrm{p}}^{(1)}=\left[\frac{\left(c_{\mathrm{e} \|}^{2}-c_{\mathrm{T} 0}^{2}\right)\left(v_{\mathrm{Ae}}^{* 2}-c_{\mathrm{T} 0}^{2}\right)}{\left(c_{\mathrm{e} \perp}^{2}+v_{\mathrm{Ae}}^{* 2}\right)\left(c_{\mathrm{Te}}^{2}-c_{\mathrm{T} 0}^{2}\right)}\right]^{1 / 2}$.

We can see that this mode propagates with a phase speed close to the internal cusp speed for wavelengths larger than the width of the tube provided $c_{\mathrm{e} \|}<c_{\mathrm{T} 0}$ and $|k| R<1 / \lambda_{\mathrm{p}}^{(1)}$.

In the thin flux tube approximation there are no fast sausage modes. Considering now the other extreme, when the wavelength of the waves is much shorter than the width of the tube (wide tube) the slow sausage modes will propagate practically without dispersion similar to waves propagating at a magnetic interface and they tend to be parallel to the horizontal axis as shown in Fig. 1.

In addition to body sausage modes, coronal plumes can also support body kink modes. In the thin flux tube approximation, slow body kink modes propagate almost without dispersion (Fig. 1). In the limit of wide tube, the dispersion relation of body kink modes is given by

$\frac{k^{2} v_{\mathrm{A} 0}^{* 2}-\omega^{2}}{k^{2} v_{\mathrm{Ae}}^{* 2}-\omega^{2}}=\mathcal{D} \frac{n_{0}}{m_{\mathrm{e}}} \tan \left(n_{0} R\right)$,

where we used an asymptotic form of Bessel functions (Abramowitz \& Stegun 1967). Comparing this form with the results obtained by Edwin \& Roberts (1983) we can see that the dispersion relation describing body kink modes in a wide tube is similar to body kink modes in a slab embedded in a

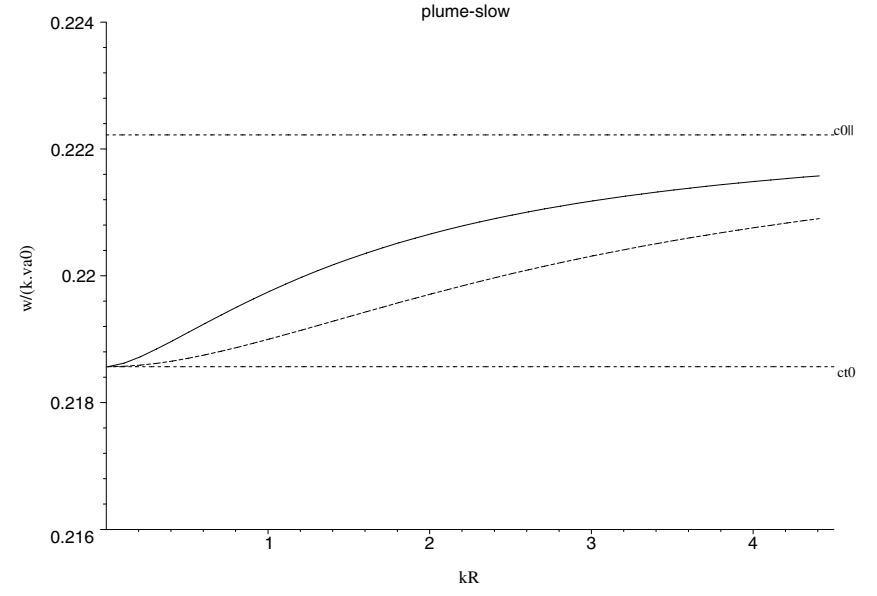

Fig. 1. Dispersion curves of slow body modes in a double-polytropic plasma tube modelling an anisotropic coronal plume: $c_{\mathrm{S} \|}^{(0)}=$ $175 \mathrm{~km} \mathrm{~s}^{-1}, c_{\mathrm{S} \perp}^{(0)}=180 \mathrm{~km} \mathrm{~s}^{-1}, v_{\mathrm{A} 0}=788 \mathrm{~km} \mathrm{~s}^{-1}, c_{\mathrm{e} \|}=140 \mathrm{~km} \mathrm{~s}^{-1}$, $c_{\mathrm{e} \perp}=160 \mathrm{~km} \mathrm{~s}^{-1}, v_{\mathrm{Ae}}=1310 \mathrm{~km} \mathrm{~s}^{-1}$. The sausage and kink modes are represented by solid (-) and dashed line (- - ), respectively. The characteristic speeds are expressed in units of the internal Alfvén speed.

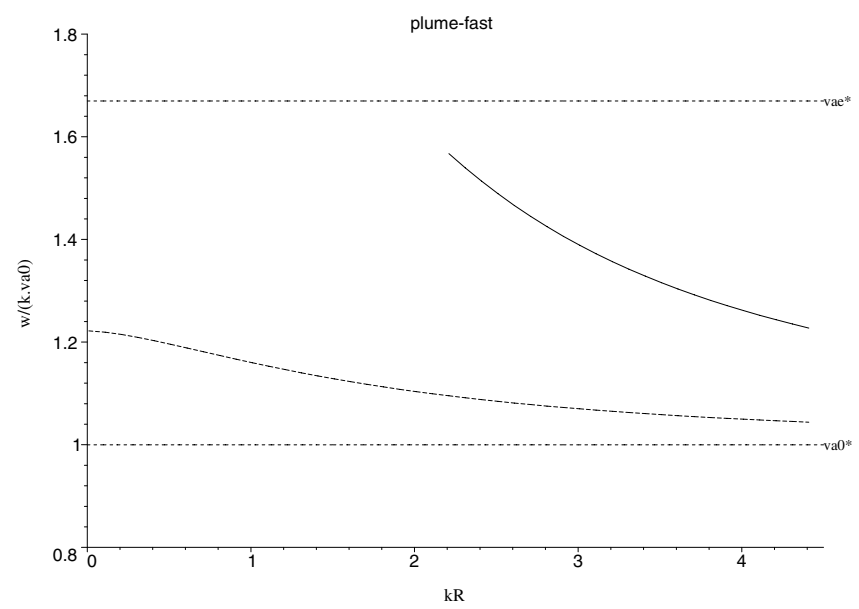

Fig. 2. The same as in Fig. 1 but for fast body waves.

magnetic environment. The cut-off value of fast modes is defined by the condition

$(k R)_{\mathrm{c}}=n \pi / \lambda_{\mathrm{p}}^{(2)}$

where

$\lambda_{\mathrm{p}}^{(1)}=\left\{-\frac{\left(v_{\mathrm{A} 0}^{* 2}-v_{\mathrm{Ae}}^{* 2}\right)\left(c_{0 \|}^{2}-v_{\mathrm{Ae}}^{* 2}\right)}{\left(c_{0 \perp}^{2}+v_{\mathrm{A} 0}^{2}\right)\left(c_{\mathrm{T} 0}^{2}-v_{\mathrm{Ae}}^{* 2}\right)}\right\}^{1 / 2}$,

and $n=0,1,2, \ldots$ We can see that the first $(n=0)$ kink mode has no cut-off (the $c_{\mathrm{k}}$-mode in Fig. 2), while higher harmonics $(n>1)$ have a cut-off which depends on the characteristic speeds and anisotropy. The dependence of the phase velocity with respect to the dimensionless radius of the plume shows that the cut-off occurs for $k R$ of the order of unity; only those waves can propagate freely in the tube whose wavelength is shorter than the diameter of the duct. A simple analysis shows that the cut-off value increases with increasing the anisotropy. Similar to the method presented by Roberts (1981) 
and Edwin \& Roberts (1983) we obtain that in the limit of a wide tube $(k R \rightarrow \infty)$, slow body kink modes propagate with a phase speed given by

$$
\frac{\omega}{k} \approx \pm c_{\mathrm{T} 0}\left\{1+\frac{c_{0 \perp}^{4}\left(v_{\mathrm{A} 0}^{* 2}-c_{\mathrm{T} 0}^{2}\right)}{2 n^{2} \pi^{2} \gamma_{\perp}^{2} c_{\mathrm{T} 0}^{2}\left(c_{0 \perp}^{2}+v_{\mathrm{A} 0}^{2}\right)^{2}} k^{2} R^{2}\right\},
$$

and a fast body kink mode given by

$$
\frac{\omega}{k} \approx \pm v_{\mathrm{A} 0}^{*}\left\{1+\frac{n^{2} \pi^{2}\left(c_{0 \perp}^{2}+v_{\mathrm{A} 0}^{2}\right)\left(v_{\mathrm{A} 0}^{* 2}-c_{\mathrm{T} 0}^{2}\right)}{2 v_{\mathrm{A} 0}^{* 2}\left(v_{\mathrm{A} 0}^{* 2}-c_{0 \|}^{2}\right) k^{2} R^{2}}\right\} .
$$

Since the plasma-beta is much less than unity, the results presented so far for slow waves do not differ too much from the results by Edwin \& Roberts (1983). Fast waves, instead, propagating with the modified Alfvén speed have a phase speed which can be very different from its isotropic counterpart.

Before we continue analyzing the properties of ducted waves in anisotropic plasmas, we should mention that there is another family of waves called backward waves which propagate in the opposite direction but since the equilibrium is static, their phase velocity dependence upon the dimensionless parameter $k R$ is symmetric with respect to the horizontal axis. In the next two cases we are going to discuss, the symmetry is broken, the forward and backward propagating waves will not be symmetric any longer. However, for the sake of simplicity we are going to consider only forward propagating waves, the complicated interaction of forward and backward propagating waves being left as a subject of further investigations.

Another factor which can influence the variation of phase velocity is the degree of anisotropy, $\Gamma$. In the dispersion relation given by Eqs. (13) and (14) there are two anisotropy factors, describing the anisotropy inside and outside the magnetic tube. In order to find out the effect of anisotropy on propagation of compressional waves, we consider first the effect of the internal anisotropy and study the change in the phase speed when $\Gamma_{0}$ is varied between -2 and 1 . If the anisotropy factor was larger than 1, the corresponding modified Alfvén speed would be imaginary and this would lead to the aforementioned firehose instability. Numerical investigation shows that in the long wavelength approximation, slow waves are very weakly influenced by the change in the internal anisotropy, practically staying almost constant. In contrast, fast waves are strongly influenced. The phase speed decreases as the anisotropy factor increases. The change in phase speed decreases with decreasing the wavelength. A completely new behaviour appears for large values of $k R$. While slow waves show again little variation with respect to the anisotropy factor, fast waves have a very steep variation. Figure 3 shows the dependence of the phase speed of fast magnetoacoustic waves propagating with phase speeds between the two modified Alfvén speeds with respect to the internal anisotropy factor. While the phase speed of kink modes decreases with increasing the anisotropy factor, sausage modes have a "peak" in their dependence. This is due to the fact that the ratio $J_{0}^{\prime}(x) / J_{0}(x)$ behave asymptotically as $\tan (x)+\cot (x)$. The peak in the variation of the phase speed is in those points where the $\cot (x)$ is singular. Kink modes do not show this behaviour since their singularities are shifted and are out of our studied range.

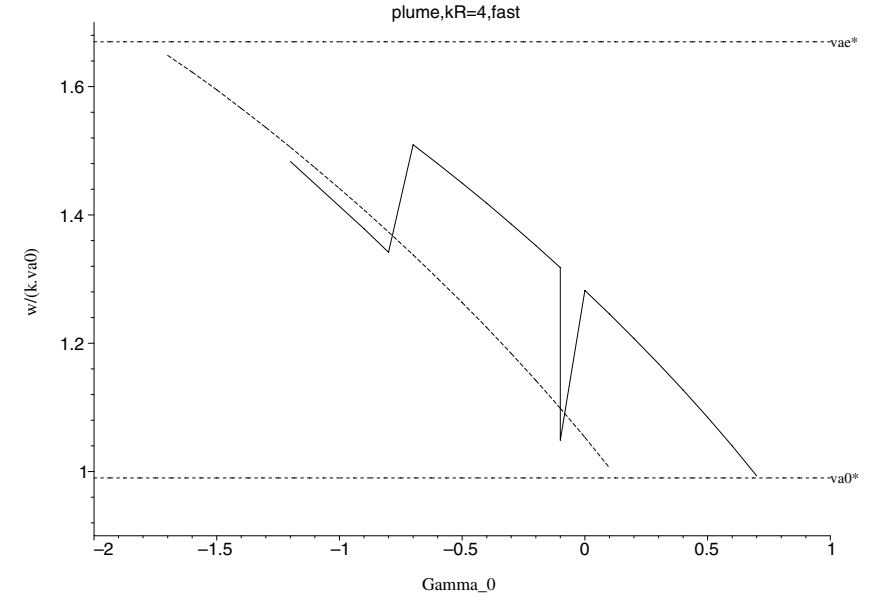

Fig. 3. Variation of phase speed for fast magnetoacoustic waves with respect to the internal anisotropy factor for $k R=4$. $\Gamma_{0}$ takes value between -2 and 1 . The sausage and kink modes are represented by solid (-) and dashed line (- - -), respectively. The characteristic speeds are expressed in units of Alfvén speed.

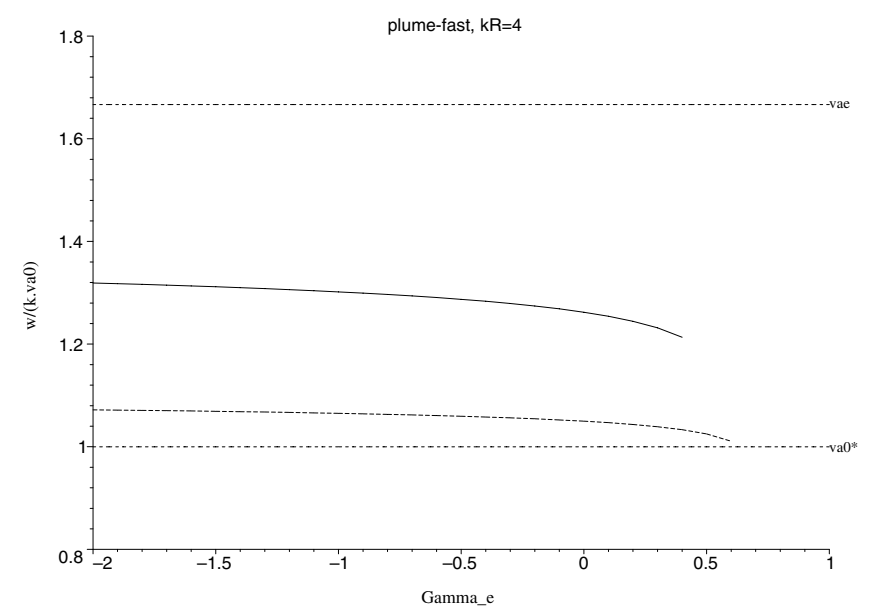

Fig. 4. The same as in Fig. 3 but phase speed is plotted with respect to the external anisotropy factor.

For small and intermediate values of $k R$ the change in the phase speed with respect to the external anisotropy factor is the same as before, with fast sausage modes declining faster then kink modes. For large values of the dimensionless quantity $k R$ the evolution of slow waves is similar to the variation found in the case of the internal anisotropy. In the case of fast waves, the dependence is shown in Fig. 4. As we can see both modes have a fairly constant variation and decay slowly at the end of their range.

\subsection{Ducted waves in the geomagnetic tail}

The existence of an extended geomagnetic tail as a waveguide has been appreciated for more than three decades (McClay \& Radoski 1967; Patel 1968; Siscoe 1969; McKenzie 1970 and more recently by Edwin et al. 1986; Walker et al. 1992; Wright 1994; Rickard \& Wright 1994; Allan \& Wright 1998; Taroyan \& Erdélyi 2003; Erdélyi \& Taroyan 2003). The magnetotail is important to energy transport between the solar wind and the 
inner magnetosphere. Ultra-low-frequency (ULF) waves can be generated by the Kelvin-Helmholtz instability on the magnetopause or in the plasma mantle (Smith et al. 1986), by plasma sheet thinning followed by expansion during substorms when the field direction changes from tail-like to dipole-like (Meng et al. 1974), or even by a flapping motion of the tail in the solar wind (Ershkovich 1972).

Observations of fluctuations or noise in the ULF band within the magnetotail have been reported by, e.g. Elphinstone et al. (1995) using IMP 8 observations of compressional waves propagating Earthward from a source situated in the tail. Later, Ables et al. (1996) and Wolfe et al. (1997) presented groundbased magnetometer observations of $\mathrm{mHz}$ frequency ULF waves in the southern polar cap, which should map to open field lines in the southern magnetotail lobe. We should note here, that the observation of Alfvén waves in the magnetotail is by far more easier due to their guided nature and their lack of attenuation. Using typical values for the geomagnetic tail ( $n \approx 10^{-2} \mathrm{~cm}^{-3}, T \approx 5.8 \times 10^{5} \mathrm{~K}$ ) we obtain that the plasma in the geomagnetic tail can be considered anisotropic for waves with frequencies much larger than $4 \times 10^{-8} \mathrm{~Hz}$.

We model the geomagnetic tail as a long cylinder with a radius $R\left(\sim 20 R_{\mathrm{E}}\right)$, outside of which flows a hot magnetized plasma that represents the shocked solar wind. Inside the tube the plasma is at rest and outside we assume that it flows with the velocity $\boldsymbol{u}_{\mathrm{e}}$ parallel to the equilibrium magnetic field. In this case, the dispersion relation for surface and body waves is similar to the previous case, but now the modified external magnetoacoustic parameter, $m_{\mathrm{e}}$, is

$m_{\mathrm{e}}^{2}=\frac{\left(k^{2} c_{\mathrm{e} \|}^{2}-\Omega^{2}\right)\left[k^{2} v_{\mathrm{Ae}}^{* 2}-\Omega^{2}\right]}{\left(c_{\mathrm{e} \perp}^{2}+v_{\mathrm{Ae}}^{2}\right)\left(k^{2} c_{\mathrm{Te}}^{2}-\Omega^{2}\right)}$,

where $\Omega=\omega-k u_{\mathrm{e}}$ denotes the Doppler-shifted frequency. We suppose that inside the cylinder the plasma- $\beta$ is much smaller than one (this is a reasonable assumption except in the vicinity of the neutral sheet) and outside the magnetic pressure dominates the kinetic pressure, i.e. the plasma- $\beta$ is larger than one. Bearing this in mind we suppose that the ordering of the characteristic speeds is such that $c_{\mathrm{T} 0}<c_{0 \|}<c_{\mathrm{Te}}+u_{\mathrm{e}}<v_{\mathrm{Ae}}^{*}+u_{\mathrm{e}}<$ $c_{\mathrm{e} \|}+u_{\mathrm{e}}<v_{\mathrm{A} 0}^{*}$.

Due to the presence of the steady equilibrium flow in the external region the limits where waves are still trapped inside the tube are affected by the considered flow but the width of the interval remains constant. Accordingly, the condition $m_{\mathrm{e}}^{2}>0$ means that the phase velocity of the waves must either be in the interval $\omega / k<c_{\mathrm{Te}}+u_{\mathrm{e}}$ or $v_{\mathrm{Ae}}^{*}+u_{\mathrm{e}}<\omega / k<c_{\mathrm{e} \|}+u_{\mathrm{e}}$.

Similar to the previous case, we are going to investigate the dispersion relations (13)-(14) for sausage $(n=0)$ and kink $(n=1)$ modes. Numerical investigation for wave propagation in the geomagnetic tail shows that for the given ordering of the characteristic speeds, only fast waves are able to propagate in this structure (see Fig. 5).

In contrast to the previous case, here we can have surface waves. Due to the particular ordering of the characteristic speeds, numerical investigations show that the geomagnetic tail can support fast surface modes only in the inter$\operatorname{val}\left(v_{\mathrm{Ae}}^{* 2}+u_{\mathrm{e}}, c_{\mathrm{e} \|}+u_{\mathrm{e}}\right)$. Because these waves propagate with

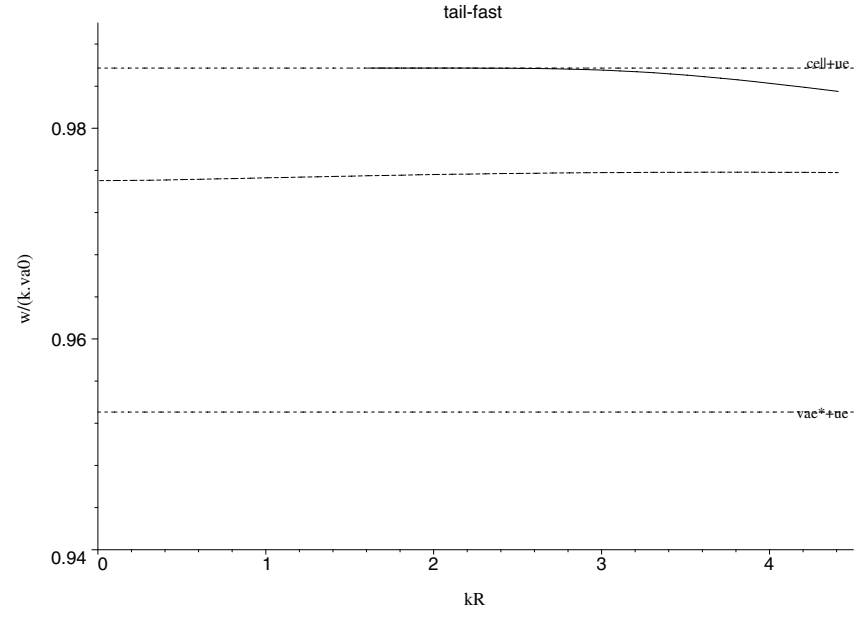

Fig. 5. Dispersion curves of fast surface modes in a double-polytropic plasma tube modelling an anisotropic geomagnetic tail, for $c_{0 \|}=$ $70 \mathrm{~km} \mathrm{~s}^{-1}, c_{\mathrm{e} \|}=250 \mathrm{~km} \mathrm{~s}^{-1}, v_{\mathrm{A} 0}^{*}=700 \mathrm{~km} \mathrm{~s}^{-1}, v_{\mathrm{Ae}}^{*}=210 \mathrm{~km} \mathrm{~s}^{-1}$, $\gamma_{\|}=1.2, \gamma_{\perp}=1.6, u_{\mathrm{e}}=450 \mathrm{~km} \mathrm{~s}^{-1}$. The sausage and kink modes are represented by solid (-) and dashed line (- - ), respectively. The characteristic speeds are expressed in units of Alfvén speed.

a phase speed close to one of the external speed, the dispersion relation in the long wavelength limit becomes transcendental, and the solutions are given by numerical investigation.

Due to the asymptotic behaviour of $K$ and $I$ functions for large arguments, surface sausage and kink modes in a wide tube behave the same and their dispersion relation is given by

$\left(k^{2} v_{\mathrm{A} 0}^{* 2}-\omega^{2}\right) m_{\mathrm{e}}+\mathcal{D}\left(k^{2} v_{\mathrm{Ae}}^{* 2}-\Omega^{2}\right) m_{0}=0$.

This dispersion relation does not contain information about the size of structure, so these waves are not dispersive. This relation can be obtained in the context of waves at a single interface, so fast surface (sausage and kink) waves propagating in a wide magnetic tube behave like waves at a single magnetic interface.

Similar to surface sausage modes, in the long wavelength approximation, there are also surface kink modes. Due to the behavior of the ratios $K_{1}(x)^{\prime} / K_{1}(x)$ and $I_{1}(x)^{\prime} / I_{1}(x)$ we can conclude that only fast kink surface waves are allowed to propagate in these structures with a phase speed close to the Doppler shifted modified Alfvén speed

$\frac{\omega}{k} \approx\left(v_{\mathrm{Ae}}^{*} \pm u_{\mathrm{e}}\right) \sqrt{\frac{\left(c_{0 \perp}^{2}+v_{\mathrm{A} 0}^{2}\right)\left[c_{\mathrm{T} 0}^{2}-\left(v_{\mathrm{Ae}}^{*}+u_{\mathrm{e}}\right)^{2}\right]}{\mathcal{D} v_{\mathrm{Ae}}^{* 2}\left[c_{0 \|}^{2}-\left(v_{\mathrm{Ae}}^{*}+u_{\mathrm{e}}\right)^{2}\right]}} \frac{1}{k R}$,

provided $c_{0 \|}<v_{\mathrm{Ae}}^{*}+u_{\mathrm{e}}$.

If the phase speed of the waves is in the interval $\left(c_{\mathrm{T} 0}, c_{0 \|}\right)$, body waves will propagate in the magnetic cylinder. Since the two characteristic speeds are very close to each other and the internal plasma beta is very small, these waves are propagating in a very narrow band. There will be slow body sausage modes propagating with a phase speed

$\frac{\omega}{k} \approx \pm c_{\mathrm{T} 0}$

$$
\times\left\{1+\frac{\mathcal{D}\left[\left(c_{\mathrm{T} 0}-u_{\mathrm{e}}\right)^{2}-v_{\mathrm{Ae}}^{* 2}\right] c_{0 \perp}^{4}}{4 \gamma_{\perp}^{2}\left(c_{0 \perp}+v_{\mathrm{A} 0}^{2}\right)^{2} c_{\mathrm{T} 0}^{2}} k^{2} R^{2} \ln \left(\lambda_{\mathrm{t}}^{(1)}|k| R\right)\right\},
$$


provided $v_{\mathrm{Ae}}^{*}<\left|c_{\mathrm{T} 0}-u_{\mathrm{e}}\right|$ and $k R>1 / \lambda_{\mathrm{t}}^{(1)}$, with

$\lambda_{\mathrm{t}}^{(1)}=\left\{\frac{\left[c_{\mathrm{e} \|}^{2}-\left(c_{\mathrm{T} 0}-u_{\mathrm{e}}\right)^{2}\right]\left[v_{\mathrm{Ae}}^{* 2}-\left(c_{\mathrm{T} 0}-u_{\mathrm{e}}\right)^{2}\right]}{\left(c_{\mathrm{e} \perp}^{2}+v_{\mathrm{Ae}}^{2}\right)\left[c_{\mathrm{Te}}^{2}-\left(c_{\mathrm{T} 0}-u_{\mathrm{e}}\right)^{2}\right]}\right\}^{1 / 2}$.

In addition, we could have fast body sausage modes propagating with a phase speed given by

$$
\begin{aligned}
\frac{\omega}{k} \approx & \pm c_{0 \|} \\
& \times \sqrt{\frac{2 c_{0 \perp}^{4}}{\mathcal{D} \gamma_{\perp}^{2}\left[v_{\mathrm{Ae}}^{2}-\left(c_{0 \|}-u_{\mathrm{e}}\right)^{2}\right] c_{0 \|}^{2} \ln \left(\lambda_{\mathrm{t}}^{(2)}|k| R\right)}} \frac{1}{k R},
\end{aligned}
$$

provided (i) $v_{\mathrm{Ae}}^{*}<\left|c_{0 \|}-u_{\mathrm{e}}\right|<c_{\mathrm{e} \|}$ and $k R<1 / \lambda_{\mathrm{t}}^{(2)}$ or (ii) $c_{\mathrm{Te}}>\left|c_{0 \|}-u_{\mathrm{e}}\right|$ and $k R>1 / \lambda_{\mathrm{t}}^{(2)}$. In Eq. (26) $\lambda_{\mathrm{t}}^{(2)}$ is defined as

$\lambda_{\mathrm{t}}^{(2)}=\left\{\frac{\left[c_{\mathrm{e} \|}^{2}-\left(c_{0 \|}-u_{\mathrm{e}}\right)^{2}\right]\left[v_{\mathrm{Ae}}^{* 2}-\left(c_{0 \|}-u_{\mathrm{e}}\right)^{2}\right]}{\left(c_{\mathrm{e} \perp}^{2}+v_{\mathrm{Ae}}^{2}\right)\left[c_{\mathrm{Te}}^{2}-\left(c_{0 \|}-u_{\mathrm{e}}\right)^{2}\right]}\right\}^{1 / 2}$.

In the wide tube limit, sausage body waves propagate without dispersion, i.e. similar to waves at a magnetic interface. Due to the behaviour of the Bessel functions for small arguments (long wavelength approximation) there are no body kink modes for thin tubes. For large arguments, the treatment of these waves is similar to the one presented in the case of waves in coronal plumes. Accordingly, we have slow waves whose phase speed is given by

$\frac{\omega}{k} \approx \pm c_{\mathrm{T} 0}\left\{1+\frac{c_{0 \perp}^{4}\left(v_{\mathrm{A} 0}^{* 2}-c_{\mathrm{T} 0}^{2}\right)}{2 \gamma_{\perp}^{4} n^{2} \pi^{2}\left(c_{0 \perp}^{2}+v_{\mathrm{A} 0}^{2}\right) c_{\mathrm{T} 0}^{2}} k^{2} R^{2}\right\}$.

Considering typical values for the geomagnetic tail $\left(c_{0 \|}=\right.$ $70 \mathrm{~km} \mathrm{~s}^{-1}, c_{\mathrm{e} \|}=250 \mathrm{~km} \mathrm{~s}^{-1}, v_{\mathrm{A} 0}^{*}=700 \mathrm{~km} \mathrm{~s}^{-1}, v_{\mathrm{Ae}}^{*}=$ $210 \mathrm{~km} \mathrm{~s}^{-1}$ ) we obtain a single fast sausage surface mode propagating in the interval $\left(c_{0 \|}, c_{\mathrm{Te}}+u_{\mathrm{e}}\right)$ whose phase speed appears for $k R>1$ and this mode is practically independent of dispersion. In the interval $\left(v_{\mathrm{Ae}}^{*}+u_{\mathrm{e}}, c_{\mathrm{e} \|}+u_{\mathrm{e}}\right)$ fast sausage and kink modes will propagate as shown in Fig. 5.

The sausage modes start at larger wavenumbers and their phase speed decreases with increasing the dimensionless quantity, $k R$. Kink modes propagate for the whole interval, but their phase speed show little variation with respect to $k R$.

The situation is different when studying the effect of anisotropy. First, we consider the effect of the internal anisotropy. The variation is shown in Fig. 6. In contrast to the case presented before (fixed anisotropy, varying wavenumber) modes for different anisotropy can have very steep variation. Between the parallel component of the internal sound speed and the Doppler shifted external cusp speed fast sausage and kink modes are able to propagate only for positive anisotropies and here both modes' phase speed decreases drastically. In the interval $\left(v_{\mathrm{Ae}}^{*}+u_{\mathrm{e}}, c_{\mathrm{e} \|}+u_{\mathrm{e}}\right)$ fast kink modes propagate only for a very limited interval.

The variation of the phase speed with respect to the internal anisotropy in a wide magnetic tube modelling the geomagnetic tail is shown in Fig. 7. Similar to the case of thin tube the two fast waves have the same behaviour. In addition to the kink

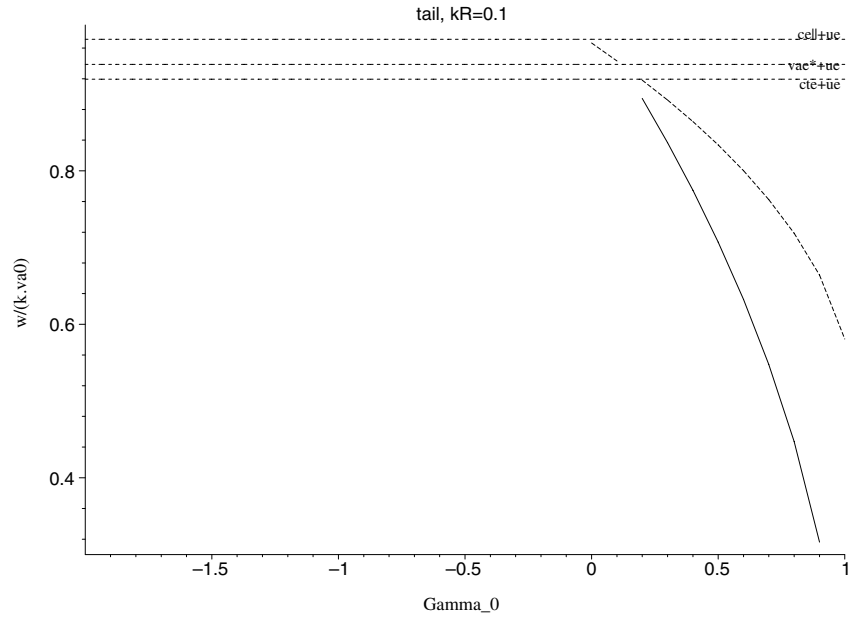

Fig. 6. Dispersion curves of surface modes in a double-polytropic plasma tube with respect to the internal anisotropy modelling an anisotropic geomagnetic tail, for $k R=0.1$.

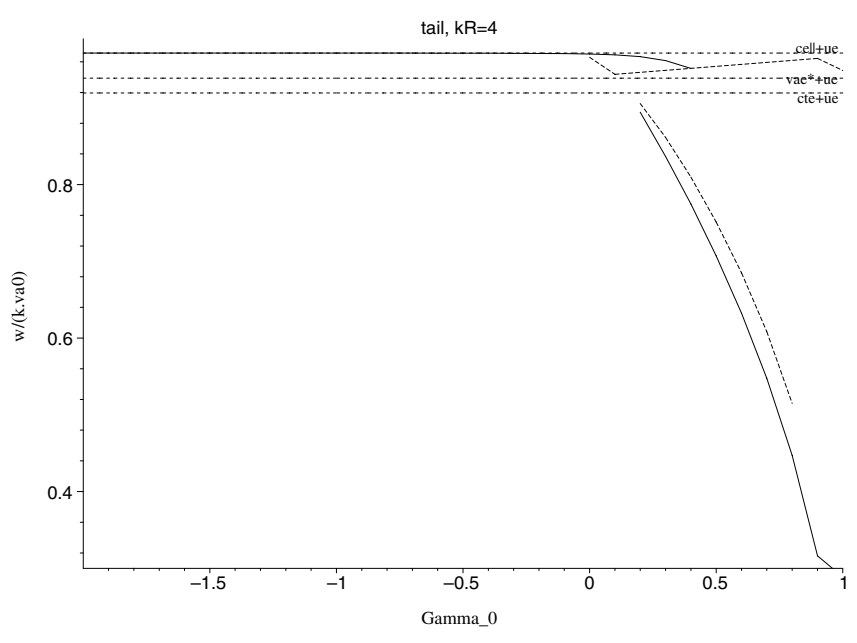

Fig. 7. The same as in Fig. 6 but for $k R=4$.

mode, in the interval $\left(v_{\mathrm{Ae}}^{*}+u_{\mathrm{e}}, c_{\mathrm{e} \|}+u_{\mathrm{e}}\right)$ we have a sausage mode whose phase speed is constant over a very large interval and decreases slowly at the end of the range.

The effect of the external anisotropy is more accentuated. For small values of $k R(\sim 0.1)$, fast surface sausage waves do not yet appear. Instead we have fast kink modes propagating in the interval $\left(v_{\mathrm{Ae}}^{*}+u_{\mathrm{e}}, c_{\mathrm{e} \|}+u_{\mathrm{e}}\right)$. These modes appear only for $\Gamma_{\mathrm{e}}>$ 0.1 . Body sausage and kink modes propagate for a very limited anisotropy $(-2,-1.6)$ where they are not dispersive. If $k R$ takes very large values $(\sim 4)$, the possible propagation modes are shown in Fig. 8. Fast sausage and kink modes appear in the interval $\left(c_{0 \|}, c_{\mathrm{Te}}+u_{\mathrm{e}}\right)$. Sausage modes appear when $\Gamma_{\mathrm{e}}>0$ and kink modes propagate only if the external anisotropy is in the interval $(0.8,1)$. In the interval $\left(v_{\mathrm{Ae}}^{*}+u_{\mathrm{e}}, c_{\mathrm{e} \|}+u_{\mathrm{e}}\right)$ we can have fast surface sausage and kink modes propagating for positive anisotropy only and both modes have a decreasing phase speed with respect to the external anisotropy factor. Similar to waves in the long wavelength limit, body waves can propagate with very small phase speed (9\% of the internal Alfvén speed) for $\Gamma_{\mathrm{e}}=(-2,-1.6)$ with very weak dispersion. These results clearly show that the effect of the anisotropy is important; for 


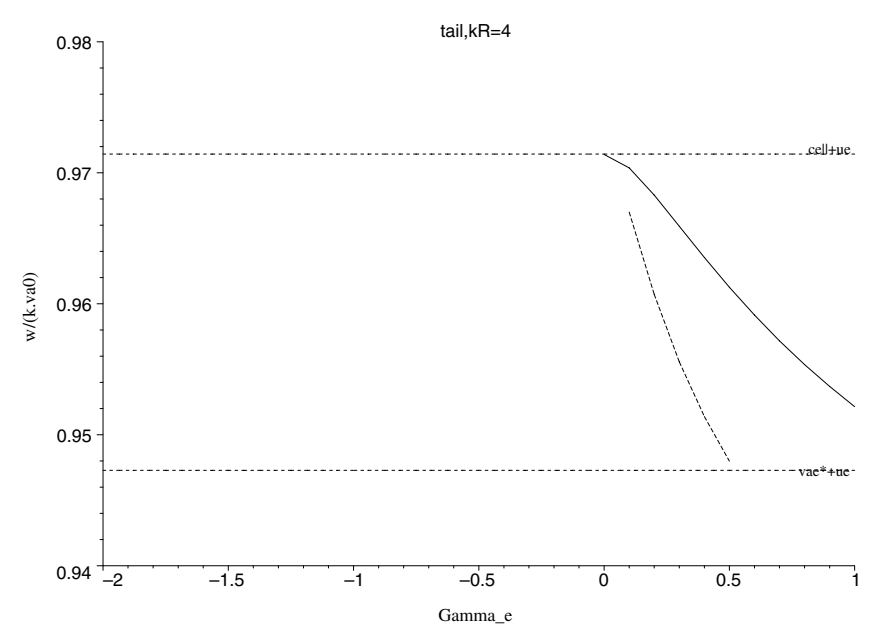

Fig. 8. Dispersion curves of fast surface modes in a double-polytropic plasma tube with respect to the external anisotropy modelling an anisotropic geomagnetic tail for $k R=4$.

certain values new modes can appear although the variation of their phase speed is not too large.

Now let us investigate the role of the equilibrium flow on the wave propagation. It is obvious that in this case the propagation windows in which waves propagate will move according to the variation of the flow. However, to emphasis the importance of an equilibrium steady flow on wave propagation we are going to plot the variation of the phase speed with respect to the equilibrium flow and the limits of propagation we choose to be at the end of flow limit. In order to find the dispersion of a wave on the figures, the propagation limits have to be shifted accordingly.

In the long wavelength approximation, slow sausage and kink surface waves can propagate in the geomagnetic tail for a very limited range of the equilibrium flow (between 170 and $195 \mathrm{~km} \mathrm{~s}^{-1}$ ) but these waves do not change their phase speed with respect to plasma flow. In this limit there are no fast surface modes. Instead there are slow body sausage and kink modes propagating in the very narrow interval $\left(c_{\mathrm{T} 0}, c_{0 \|}\right)$. Sausage modes propagate provided $u_{\mathrm{e}}<200 \mathrm{~km} \mathrm{~s}^{-1}$ and kink modes provided $u_{\mathrm{e}}<100 \mathrm{~km} \mathrm{~s}^{-1}$. Both modes show no variation with respect to the equilibrium flow.

In the wide tube limit, the only possible propagation mode lies in the interval $\left(c_{0 \|}, c_{\mathrm{Te}}+u_{\mathrm{e}}\right)$ and here the variation of the phase speed with respect to the equilibrium flow is practically constant.

\subsection{Ducted waves in anisotropic astrophysical jets}

Collimated outflows are quite common in astrophysics and cosmic jets. They are observed in the radio, infrared, optical, UV and X-ray parts of the spectrum, from ground and space based telescopes, most recently via the Hubble Space Telescope (HST).

The presence of magnetic fields in low density extragalactic radio jets may cause a decoupling of the particle velocities parallel and perpendicular to the magnetic field direction. Consequently, our approach of anisotropic plasma with a plasma pressure can be used to describe the wave dynamics in these structures. Even though the collisional mean free path is much larger than the transverse dimensions of the jet, the assumption of a fluid model can be justified taking into account that the presence of the magnetic field constrains the motion of the particles transverse to the jet, and therefore introduces an effective mean free path, of the order of the particle gyroradius, which is much smaller than the dynamical scales of the plasma. Using typical parameters $\left(n \approx 10^{-3} \mathrm{~cm}^{-3}, T \approx 10^{3} \mathrm{~K}\right)$ we obtain that the plasma can be considered anisotropic if the frequency of the waves is much larger than $1.3 \times 10^{-7} \mathrm{~Hz}$.

We model the astrophysical jet with a magnetic cylinder in which there is a plasma flow parallel to the magnetic field $\left(\boldsymbol{u}_{0}\right)$. This tube is embedded in a magnetic-free isotropic fluid. The method of obtaining the dispersion relation is similar to the case described in details previously, but here $m_{0}^{2}$ and $m_{\mathrm{e}}^{2}$ are modified as follows

$m_{0}^{2}=\frac{\left(k^{2} c_{0 \|}^{2}-\Omega^{2}\right)\left[k^{2} v_{\mathrm{A} 0}^{* 2}-\Omega^{2}\right]}{\left(c_{0 \perp}^{2}+v_{\mathrm{A} 0}^{2}\right)\left(k^{2} c_{\mathrm{T} 0}^{2}-\Omega^{2}\right)}$,

$m_{\mathrm{e}}^{2}=\frac{k^{2} c_{\mathrm{Se}}^{2}-\omega^{2}}{c_{\mathrm{Se}}^{2}}$

where now $\Omega=\omega-k u_{0}$ is the Doppler-shifted frequency inside the jet.

For trapped waves to occur under the considered circumstances, the phase speed of the waves must be below the external sound speed. The density ratio for this configuration is given by

$\mathcal{D}=\frac{c_{0 \perp}^{2}}{c_{\mathrm{Se}}^{2}}+\frac{1}{2} \gamma_{\perp} \frac{v_{\mathrm{A} 0}^{2}}{c_{\mathrm{Se}}^{2}}$.

Since in astrophysical jets $\mathcal{D}<1$, the ordering of the characteristic speeds is $c_{\mathrm{T} 0}+u_{0}<v_{\mathrm{A} 0}^{*}+u_{0}<c_{0 \|}+u_{0}<c_{\mathrm{Se}}$. For this ordering, we can have slow surface waves in the interval below the Doppler-shifted cusp speed, slow body waves in the interval $\left(c_{\mathrm{T} 0}+u_{0}, v_{\mathrm{A} 0}^{*}+u_{0}\right)$, fast surface waves in the interval between the Doppler-shifted modified Alfvén speed and the Doppler-shifted internal parallel sound speed, and finally, fast body waves in the interval between the Doppler-shifted internal parallel sound speed and the external sound speed. All these modes exist provided $u_{0}<c_{\mathrm{Se}}-c_{0 \|}$. If $c_{\mathrm{Se}}-c_{0 \|}<u_{0}<c_{\mathrm{Se}}-v_{\mathrm{A} 0}^{*}$, fast body modes disappear. If $c_{\mathrm{Se}}-c_{\mathrm{T} 0}<u_{0}<c_{\mathrm{Se}}-v_{\mathrm{A} 0}^{*}$, fast surface modes cease to exist as well, and the only modes which still propagate are slow surface and body modes.

In Fig. 9, the dispersion curves corresponding to real conditions of astrophysical jets are shown.

Considering again the dispersion relation for surface and body waves, we have slow surface sausage modes propagating in a thin flux tube with a phase speed given by

$$
\begin{array}{r}
\frac{\omega}{k} \approx\left(u_{0} \pm c_{\mathrm{T} 0}\right)\left\{1+\frac{\mathcal{D}\left(c_{\mathrm{T} 0}+u_{0}\right)^{2} c_{0 \perp}^{4}}{4 c_{\mathrm{T} 0}^{2} \gamma_{\perp}^{2}\left(c_{0 \perp}^{2}+v_{\mathrm{A} 0}^{2}\right)^{4}} k^{2} R^{2}\right. \\
\left.\times \ln \left(\lambda_{j}^{(1)}|k| R\right)\right\},
\end{array}
$$




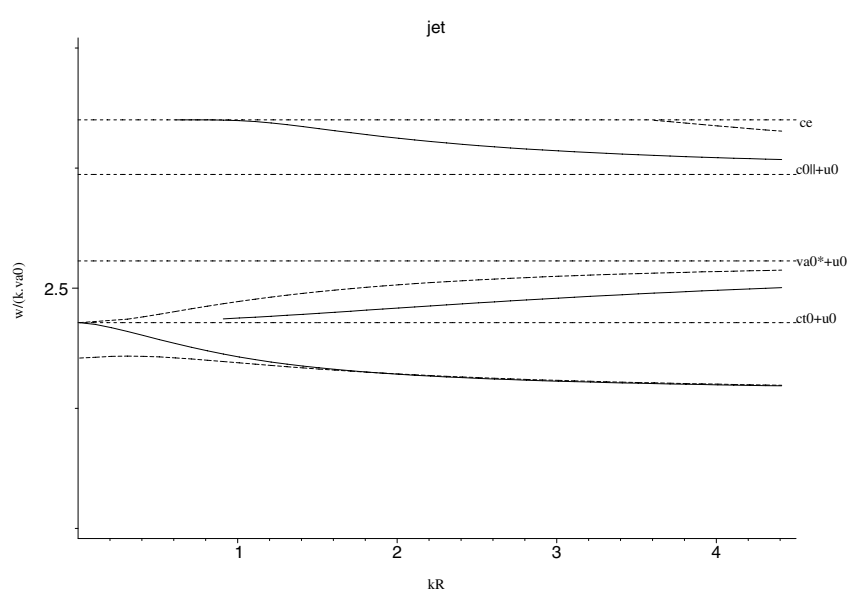

Fig. 9. Dispersion curves for sausage (full line) and kink (dotted line) modes arising in a double-polytropic plasma tube modelling an anisotropic astrophysical jet: $c_{0 \|}=480 \mathrm{~km} \mathrm{~s}^{-1}, c_{0 \perp}=520 \mathrm{~km} \mathrm{~s}^{-1}$, $v_{\mathrm{A} 0}=220 \mathrm{~km} \mathrm{~s}^{-1}, c_{\mathrm{Se}}=920 \mathrm{~km} \mathrm{~s}^{-1}, u_{0}=300 \mathrm{~km} \mathrm{~s}^{-1}$. The phase speeds are expressed in units of Alfvén speed.

provided $|k| R<1 / \lambda_{j}^{(1)}$ and

$\lambda_{j}^{(1)}=\left[1-\left(\frac{c_{\mathrm{T} 0}+u_{0}}{c_{\mathrm{Se}}}\right)^{2}\right]^{1 / 2}$

For wide tubes, slow surface sausage waves propagate without dispersion similar to waves at a magnetic interface. We found no fast surface sausage modes.

In the case of kink waves propagating in the long wavelength limit, the propagation occurs without significant dispersion and there will be a slow surface kink wave propagating with a phase speed

$\frac{\omega}{k} \approx\left(u_{0} \pm c_{\mathrm{T} 0}\right)\left\{1-\mathcal{D}^{2} \frac{\left(c_{\mathrm{T} 0}+u_{0}\right)^{2} c_{0 \perp}^{4}}{4 \gamma_{\perp}^{2}\left(c_{0 \perp}^{2}+v_{\mathrm{A} 0}^{2}\right)^{2} c_{\mathrm{T} 0}^{2}} k^{2} R^{2}\right\}$

Similar to the sausage modes, there is no fast surface kink wave propagating in the thin flux tube approximation. In the thick flux tube (short wavelength) limit we have only slow waves propagating practically without dispersion.

In addition to surface waves, body waves are propagating in the interval $\left(c_{\mathrm{T} 0}+u_{0}, v_{\mathrm{A} 0}^{*}+u_{0}\right)$ for slow waves and $\left(c_{0 \|}+u_{0}, c_{\mathrm{Se}}\right)$ for fast waves. In the thin flux tube limit, there will be slow body sausage modes propagating with a phase speed given by

$$
\begin{aligned}
\frac{\omega}{k} \approx & \left(u_{0} \pm c_{\mathrm{T} 0}\right) \\
& \times\left\{1+\frac{\mathcal{D}\left(c_{\mathrm{T} 0}+u_{0}\right)^{2} c_{0 \perp}^{4}}{4 \gamma_{\perp}^{2} c_{\mathrm{T} 0}^{2}\left(c_{0 \perp}+v_{\mathrm{A} 0}^{2}\right)^{4}} k^{2} R^{2} \ln \left(\lambda_{j}^{(1)}|k| R\right)\right\},
\end{aligned}
$$

where $\lambda_{j}^{(1)}$ is given by (32). This modes exists provided $k R>$ $1 / \lambda_{j}^{(1)}$. Bearing in mind the asymptotic behaviour of $\lambda_{j}^{(1)}$, we can easily see that this mode appears only for values of $k R$ close to 1 . In contrast to surface modes, we can have fast body sausage modes, propagating with a phase speed close to the external sound speed. Since the analytical analysis of this mode require solving a transcendental function, we investigate this

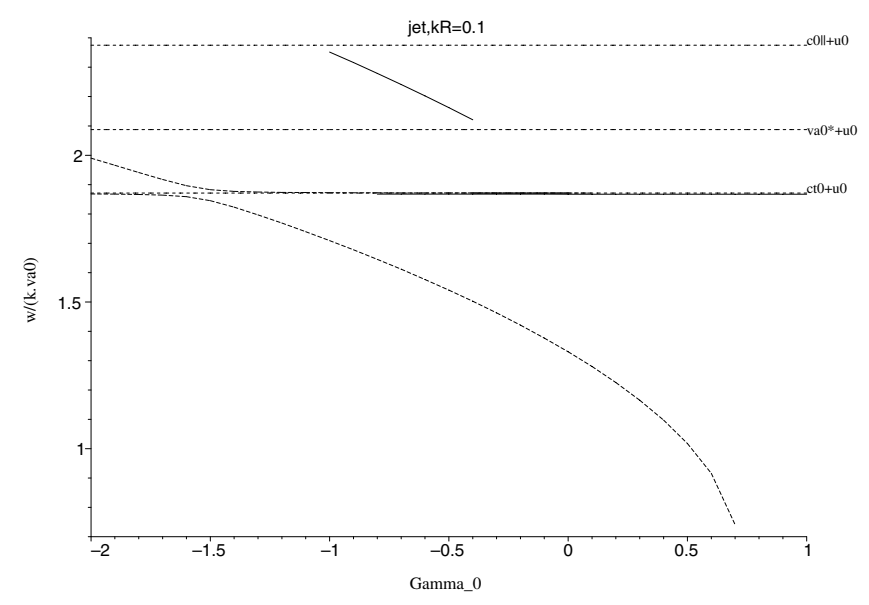

Fig. 10. Variation of the phase velocity of the waves in an anisotropic astrophysical jet with respect to the anisotropy factor for $k R=0.1$.

case numerically. In the other extreme (short wavelength) all slow surface modes propagate without dispersion.

In the case of kink modes propagating with wavelengths much larger than the radius of the duct, we obtain that slow body kink modes are able to propagate and these modes are not dispersive. Their phase speed given by

$\frac{\omega}{k} \approx\left(u_{0} \pm c_{\mathrm{T} 0}\right)\left\{1+\frac{c_{0 \perp}^{2}\left(v_{\mathrm{A} 0}^{* 2}-c_{\mathrm{T} 0}^{2}\right)}{2 \gamma_{\perp}^{2} n^{2} \pi^{2}\left(c_{0 \perp}^{2}+v_{\mathrm{A} 0}^{2}\right)^{2}} k^{2} R^{2}\right\}$.

Fast kink body modes propagate with a phase speed

$\frac{\omega}{k} \approx\left(u_{0} \pm c_{0 \|}\right)\left\{1+\frac{n^{2} \pi^{2} c_{0 \perp}^{4}}{2 \gamma_{\perp}^{2} c_{0 \|}^{2}\left(c_{0 \|}^{2}-v_{\mathrm{A} 0}^{* 2}\right)}\right\}$,

where $n$ is an integer number.

In this limit, the dispersion relation is similar to the dispersion relation found for a magnetic slab in the wide slab limit, so we can conclude that body waves in a wide tube behave similar to waves in a wide slab. The possible propagation of compressional waves in a magnetic slab can be found in, e.g. Roberts (1981) for an isotropic plasma or Ballai et al. (2002) for an anisotropic rarefied plasma.

Now, let us investigate the effect of the anisotropy on wave propagation in an astrophysical anisotropic jet. Similar to the previous cases, we vary the internal anisotropy factor between -2 and 1 for different values of the dimensionless quantity $k R$. In the thin flux tube limit the variation of the phase speed of the slow and fast waves with respect to the anisotropy factor is represented in Fig. 10. It is clear that the slow sausage surface modes are practically independent of the anisotropy for the whole length of the chosen interval. Slow kink surface modes propagate provided $\Gamma<0.7$ and they show a drastically decreasing phase speed with respect to the anisotropy factor. At the end of the interval, the phase velocity is almost three times smaller than at the beginning of the interval. Between the Doppler shifted cusp speed and the Doppler shifted modified Alfvén speed we have slow body modes. Slow sausage body modes are independent of the variation of the anisotropy and they propagate if the anisotropy factor is in the 


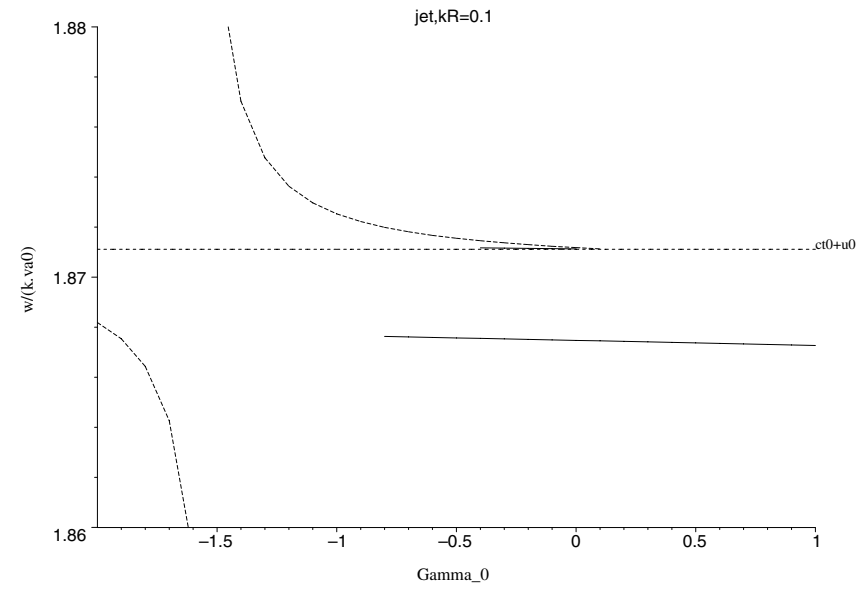

Fig. 11. Variation of the phase velocity of the waves in an anisotropic astrophysical jet with respect to the anisotropy factor for $k R=0.1$. The figure shows the zoomed region around $c_{\mathrm{T} 0}+u_{0}$.

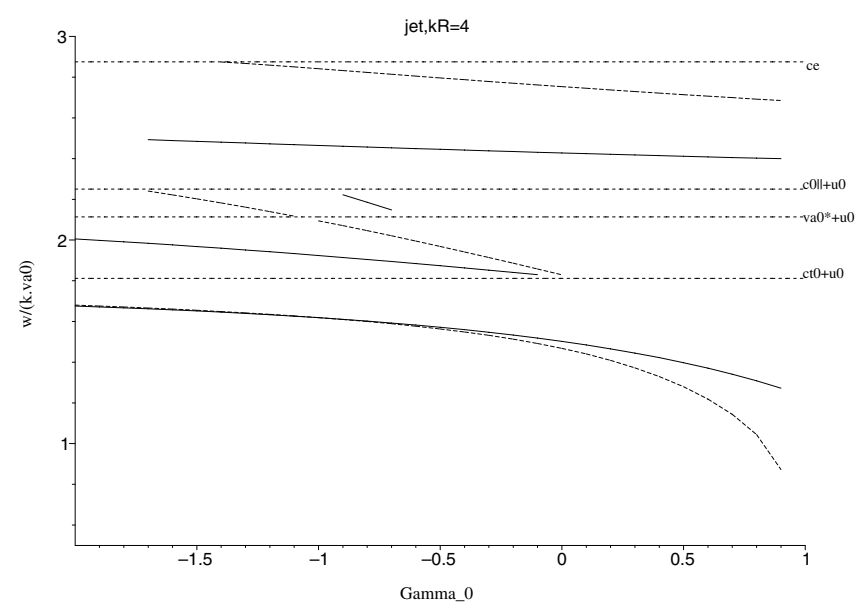

Fig. 12. The same as in Fig. 10 but for $k R=4$.

interval $(-0.4,0.2)$. The corresponding kink modes appear for $\Gamma=(-2,0.1)$ and they show little variation with respect to the anisotropy factor. Fast sausage surface modes appear in the interval $\left(v_{\mathrm{A} 0}^{*}+u_{0}, c_{0 \|}+u_{0}\right)$ and these modes have a decreasing behaviour with respect to increasing anisotropy factor. They appear only if the anisotropy is in the interval $(-1,-0.4)$. Figure 11 shows a zoom of the range around the Doppler shifted cusp speed.

Figure 12 shows the variation of the phase speed with respect to the anisotropy for a wide tube. Slow sausage and kink surface modes decrease with increasing the anisotropy factor. Slow body sausage and kink modes appear only in a limited interval and both modes show a decreasing tendency with respect to the anisotropy. In the interval $\left(v_{\mathrm{A} 0}^{*}+u_{0}, c_{0 \|}+u_{0}\right)$, fast sausage and kink surface modes have a decreasing phase speed and they disappear when $\Gamma \sim-0.7$. Fast body sausage and kink modes are present for almost all values of the anisotropy and they have a slowly decreasing tendency.

Another important parameter which should be taken into account is the strength of the flow in these astrophysical jets. Figure 13 shows the dependence of the phase speed with

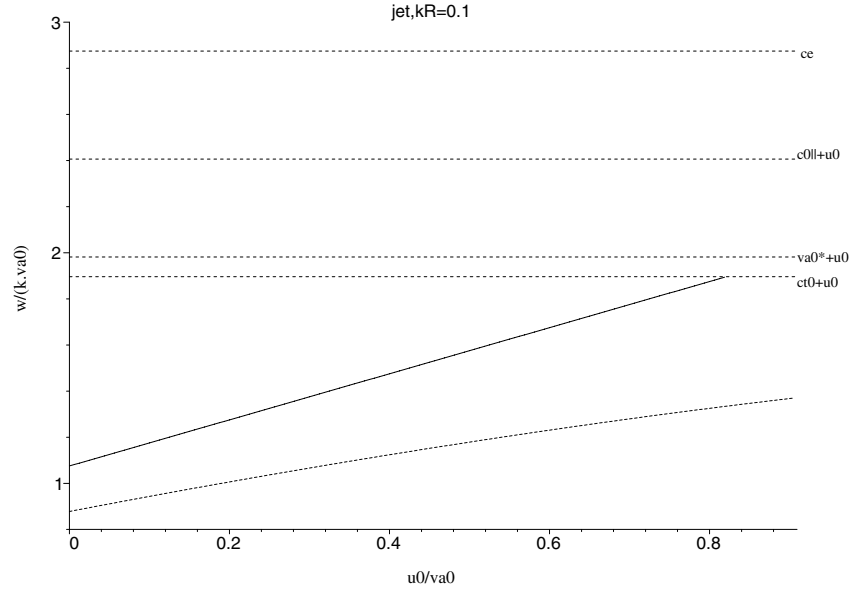

Fig. 13. Variation of the phase speed of the waves in an anisotropic astrophysical jet with respect to the equilibrium flow in the long wavelength approximation $(k R=0.1)$. The strength of the field aligned flow is varied between 0 and 300 . The characteristic velocities are the same as in the previous figures. The phase velocity of the waves is expressed in units of Alfvén speed.

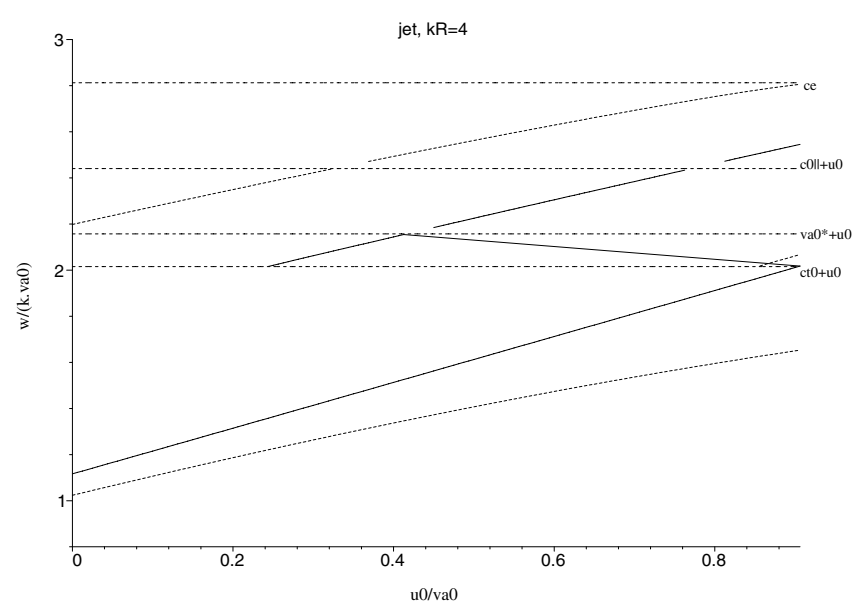

Fig. 14. The same as in Fig. 13 but for $k R=4$.

respect to the equilibrium flow when this is varied between 0 and $300 \mathrm{~km} \mathrm{~s}^{-1}$ in the long wavelength limit. In the interval $\left(0, c_{\mathrm{T} 0}+u_{0}\right)$, slow sausage and kink surface waves propagate (solid and dotted lines). In this interval, both modes' phase speed increases with respect to the equilibrium flow.

For the other extreme (wide tube), the possible modes arising in astrophysical jets when the flow strength is varied are shown in Fig. 14. Slow sausage and kink surface modes have the same behaviour as in the thin flux tube limit. Slow body sausage and kink modes propagate between $\left(c_{\mathrm{T} 0}+u_{0}, v_{\mathrm{A} 0}^{*}+u_{0}\right)$. While sausage modes appear for $u_{0}>0.24 v_{\mathrm{A} 0}$, kink modes oscillate only for very high flow speeds $\left(>0.85 v_{\mathrm{A} 0}\right)$. Fast surface sausage and kink modes propagate between the Doppler shifted modified Alfvén speed and parallel component of sound speed and both modes exhibit an increasing phase speed with increasing the equilibrium plasma flow. Fast body sausage and kink modes appear in the interval $\left(c_{0 \|}+u_{0}, c_{\mathrm{Se}}\right)$ and, again, both modes have an increasing phase speed with respect to the plasma flow. These figures clearly show that an 
equilibrium flow has a very important role in the process of wave propagation. The presence of this plasma flow can generate or stop modes propagating in an anisotropic astrophysical jet. The presence of the flow can shift the modes as Fig. 14 shows: fast surface sausage and kink modes for increasing plasma flow become fast body sausage and kink modes.

\section{Conclusions}

The present study is a natural extension of the previous studies by Hau \& Lin (1995) and Ballai et al. (2002). The study has concentrated on the behaviour of slow and fast magnetoacoustic waves propagating in a magnetic tube under different circumstances. The plasma has been considered anisotropic in the kinetic pressure. To describe the energy evolution over the two directions (parallel and perpendicular to the equilibrium magnetic field) we have used a double-polytropic law.

Firstly, we considered waves propagating in the coronal plume region. Plumes were approximated by a magnetic cylinder embedded in the magnetic interplume region. In both regions the plasma was considered anisotropic and there was no equilibrium flow taken into account. Since the plasma-beta in these structures is very small, the presented case is similar to the case first studied by Edwin \& Roberts (1983) for an isotropic plasma in coronal loops. Similar to their findings, the only kind of compressional waves able to propagate in these structures were body waves. We have shown that a change in the external anisotropy does not induce any significant change in the phase speed of the waves. In contrast, for changes in the internal anisotropy the phase speeds are strongly affected, and in general an increase in the anisotropy leads to a decrease in the phase speed of the waves.

The geomagnetic tail surrounded by the shocked solar wind can support only fast surface sausage and kink waves. In this particular situation, the variation of the internal anisotropy leads to large changes (decrease) in the phase speed of the fast waves.

The variation of the external anisotropy may lead to appearance of new modes and certain modes propagate only for a limited interval of the anisotropy and fast waves were mostly affected for short wavelengths. The propagation of linear compressional waves in the geomagnetic tail has been studied when the flow speed of the solar wind changes. Large variations in the phase speed have not been observed numerically.

Finally, the analytical results have been applied to study the possible mode propagation in anisotropic astrophysical jets. Again, the importance of the anisotropy and the equilibrium flow present in the jet have been investigated. The obtained results show that both quantities are very important; i.e. the phase speed of the waves exhibits strong variation with respect to these two parameters.

Acknowledgements. The authors acknowledge Mr. M. Homem and Y. Taroyan for the assistance with numerical part of the present paper and for providing magnetospheric data. I.B. acknowledge the financial support by PPARC (UK) and the NFS Hungary (OTKA, T043741).

\section{Appendix A: The linearized MHD equations}

The linearization of the system of Eqs. (1)-(4) yields

$$
\begin{aligned}
& \mathrm{i} \omega \rho=\frac{\rho_{0}}{r} \frac{\mathrm{d}}{\mathrm{d} r}\left(r v_{\mathrm{r}}\right)+\mathrm{i} \rho_{0}\left[\frac{m}{r} v_{\varphi}+k v_{z}\right]+v_{\mathrm{r}} \frac{\mathrm{d} \rho_{0}}{\mathrm{~d} r}, \\
& \omega B_{\mathrm{r}}=-k v_{\mathrm{r}} B_{0}, \quad \omega B_{\varphi}=-k v_{\varphi} B_{0}, \\
& \mathrm{i} \omega B_{z}=\frac{\mathrm{d}}{\mathrm{d} r}\left(v_{\mathrm{r}} B_{0}\right)+\frac{v_{\mathrm{r}} B_{0}}{r}+\frac{\mathrm{i} m}{r} B_{0} v_{\varphi},
\end{aligned}
$$

$$
\begin{aligned}
\mathrm{i} \omega p_{\perp}= & \frac{\mathrm{i} \omega c_{\mathrm{S} \perp}^{2}}{\gamma_{\perp}} \rho+\frac{\mathrm{i} \omega\left(\gamma_{\perp}-1\right) p_{\perp}^{(0)}}{B_{0}} B_{z} \\
& +v_{\mathrm{r}}\left[\frac{\mathrm{d} p_{\perp}^{(0)}}{\mathrm{d} r}-\frac{c_{\mathrm{S} \perp}^{2}}{\gamma_{\perp}} \frac{\mathrm{d} \rho_{0}}{\mathrm{~d} r}-\frac{\left(\gamma_{\perp}-1\right) p_{\perp}^{(0)}}{B_{0}} \frac{\mathrm{d} B_{0}}{\mathrm{~d} r}\right]
\end{aligned}
$$

$$
\begin{aligned}
\mathrm{i} \omega p_{\|}= & \mathrm{i} \omega c_{\mathrm{S} \|}^{2} \rho-\frac{\mathrm{i} \omega\left(\gamma_{\|}-1\right) p_{\|}^{(0)}}{B_{0}} B_{z} \\
& +v_{\mathrm{r}}\left[\frac{\mathrm{d} p_{\|}^{(0)}}{\mathrm{d} r}-c_{\mathrm{S} \|}^{2} \frac{\mathrm{d} \rho_{0}}{\mathrm{~d} r}+\frac{\left(\gamma_{\|}-1\right) p_{\|}^{(0)}}{B_{0}} \frac{\mathrm{d} B_{0}}{\mathrm{~d} r}\right],
\end{aligned}
$$

$\mathrm{i} \omega \rho_{0} v_{\mathrm{r}}=\frac{\mathrm{d} P_{\perp}}{\mathrm{d} r}-\frac{\mathrm{i} k(1-\Gamma) B_{0}}{\mu} B_{\mathrm{r}}$,

$\mathrm{i} \omega \rho_{0} v_{\varphi}=\frac{\mathrm{i} m}{r} P_{\perp}-\frac{\mathrm{i} k(1-\Gamma) B_{0}}{\mu} B_{\varphi}$

$\mathrm{i} \omega \rho_{0} v_{z}=\mathrm{i} k p_{\|}-\frac{\mathrm{i} k \Gamma B_{0}}{\mu} B_{z}-\frac{B_{\mathrm{r}}}{\mu} \frac{\mathrm{d}}{\mathrm{d} r}\left[B_{0}(1-\Gamma)\right]$,

where

$P_{\perp}=p_{\perp}+\frac{B_{0}^{2}}{2 \mu}$

is the total pressure perturbation across the magnetic field lines, $\Gamma=\left(p_{\|}^{(0)}-p_{\perp}^{(0)}\right) / v_{A}^{2} \rho_{0}$ is the anisotropy factor and $\boldsymbol{v}=\left(v_{\mathrm{r}}, v_{\phi}, v_{z}\right)$, $\boldsymbol{B}=\left(B_{\mathrm{r}}, B_{\phi}, B_{z}\right)$ are the perturbations of the velocity and magnetic field.

\section{References}

Ables, S. T., Fraser, B. J., Hansen, H. J., Menk, F. W., \& Morris, R. J. 1996, Aust. Natl. Antarct. Res. Exped., Melbourne, Victoria, ANARE Res. Notes, 95, 112,

Abraham-Shrauner, B. W. 1967, J. Plasma Phys., 1, 361

Abramowitz, M., \& Stegun, I. A. 1967, Handbook of Mathematical Functions (Dover)

Allan, W., \& Wright, A. N. 1998, J. Geophys. Res., 103, 2359

Ballai, I, Erdélyi, R., \& Roberts, B. 2002, Ann. Geophys, 20, 1553

Ballai, I., Erdélyi, R., Voitenko, Y., \& Goossens, M. 2002, Phys. Plasmas, 9, 2593

Banerjee, D., O'Shea, E., Doyle, J. G., \& Goossens, M. 2001, A\&A, 377, 691

Chew, G. F., Goldberger, M. L., \& Low, F. E. 1956, Proc. Roy. Soc. London, Ser A, 236, 112

DeForest, C. E., \& Gurman, J. B. 1998, ApJ, 501, L217 
Edwin, P. M., \& Roberts, B. 1983, Sol. Phys., 76, 239

Edwin, P. M., Roberts, B., \& Hughes, W. J. 1986, Geophys. Res. Lett., 13,373

Elphinstone, R. D., Hearn, D. J., \& Cogger, L. L. 1995, J. Geophys. Res., 100, 12093

Erdélyi, R., \& Taroyan, Y. 2003, J. Geophys. Res., 108(A1), 1043

Ershakovich, A. I., Nusinov, A. A., \& Chernikov, A. A. 1972, J. Geophys. Res., 77, 6907

Farrugia, C. J., Erkaev, N. V., Vogl, D. F., et al. 2001, J. Geophys. Res., 106,29373

Hau, L.-N., \& Lin, C.-A. 1995, Phys. Plasmas, 2, 294

Homem, M. T., Erdélyi, R., \& Ballai, I. 2003, Sol. Phys., in press Joarder, P. S. 2002, A\&A, 384

Kato, Y., Tajiri, M., \& Taniuti, T. 1966, J. Phys. Soc. Jpn, 66, 765

Kohl, J. L., Noci, G., \& Antonucci, E. 1998, ApJ, 501, L127

McClay, J. R., \& Radoski, H. R. 1967, J. Geophys. Res., 72, 4525

McKenzie, J. F. 1970, J. Geophys. Res., 75, 5331

Meng, C. I., \& Colburn, D. S. 1974, J. Geophys. Res., 79, 1831

Nakariakov, V. M., \& Oraevsky, V. N. 1995, Sol. Phys., 158, 29

Nakariakov, V. M., \& Roberts, B. 1995, Sol. Phys., 195, 213
Ofman, L., Romali, M., Polleto, G., Noci, G., \& Kohl, J. L. 2000, ApJ, 529,592

Parker, E. N. 1958, Phys. Rev., 109, 1874

Patel, V. L. 1968, Phys. Lett, 26A, 596

Rickard, G. J., \& Wright, A. N. 1994, J. Geophys. Res., 99, 13455

Roberts, B. 1981, Sol. Phys., 69, 39

Siscoe, G. L. 1969, J. Geophys. Res., 74, 6482

Smith, R. A., Goertz, C. K., \& Grossmann, W. 1986, Geophys. Res. Lett., 13, 1380

Spruit, H. C. 1982, Sol. Phys., 75, 3

Srivastava, K. M. 1977, A\&A, 54, 195

Taroyan, Y., \& Erdélyi, R. 2003, Phys. Plasmas, 10, 266

Trussoni, E., Massaglia, S., Bodo, G., \& Ferrari, A. 1988, MNRAS, 234, 539

Walker, A. D. M., Ruohoniemi, J. M., Baker, K. B., Greenwald, R. A., \& Samson, J. C. 1992, J. Geophys. Res., 97, 12187

Wilson, P. R. 1980, A\&A, 87, 121

Wolfe, A., Lanzerotti, L. J., Maclennan, C. G., \& Weatherwax, A. T. 1997, EOS Trans. AGU, 78, S227

Wright, A. N. 1994, J. Geophys. Res., 99, 159 